\title{
A Highly Accurate Regular Domain Collocation Method for Solving Potential Problems in the Irregular Doubly Connected Domains
}

\author{
Zhao-Qing Wang, ${ }^{1}$ Shuchen $\mathrm{Li}^{2}{ }^{2}$ Yang Ping, ${ }^{2}$ Jian Jiang, ${ }^{1}$ and Teng-Fei $\mathrm{Ma}^{2}$ \\ ${ }^{1}$ Institute of Engineering Mechanics, Shandong Jianzhu University, Jinan 250101, China \\ ${ }^{2}$ Geotechnical and Structural Engineering Research Center, Shandong University, Jinan 250061, China
}

Correspondence should be addressed to Shuchen Li; shuchenli@sdu.edu.cn

Received 15 December 2013; Accepted 8 March 2014; Published 23 April 2014

Academic Editor: Miaojuan Peng

Copyright (C) 2014 Zhao-Qing Wang et al. This is an open access article distributed under the Creative Commons Attribution License, which permits unrestricted use, distribution, and reproduction in any medium, provided the original work is properly cited.

\begin{abstract}
Embedding the irregular doubly connected domain into an annular regular region, the unknown functions can be approximated by the barycentric Lagrange interpolation in the regular region. A highly accurate regular domain collocation method is proposed for solving potential problems on the irregular doubly connected domain in polar coordinate system. The formulations of regular domain collocation method are constructed by using barycentric Lagrange interpolation collocation method on the regular domain in polar coordinate system. The boundary conditions are discretized by barycentric Lagrange interpolation within the regular domain. An additional method is used to impose the boundary conditions. The least square method can be used to solve the overconstrained equations. The function values of points in the irregular doubly connected domain can be calculated by barycentric Lagrange interpolation within the regular domain. Some numerical examples demonstrate the effectiveness and accuracy of the presented method.
\end{abstract}

\section{Introduction}

In physics, mechanics, and other disciplines, Poisson equation or Laplace equation is used as the governing equation to describe electric potential, temperature, and many other physical quantities. The functions satisfying Laplace equation are called potential functions and the problems of electric potential, temperature, and so forth are also known as potential problems. In engineering problems, we need to inevitably solve potential problems in complex regions and the doubly connected domain composed of two closed curves is a typical complex region. Therefore, how to precisely solve potential problems in complex regions is an important issue in the field of numerical calculation.

The finite element method (FEM) is an effective numerical method for solving potential problems in complex domains $[1,2]$. However, to fit boundaries of complex regions and improve the calculation accuracy, FEM needs to divide dense elements, reducing the computational efficiency. The meshless methods, such as complex variable element-free Galerkin method [3-6], interpolating boundary element-free method [7], improved element-free Galerkin method [8-10], complex variable reproducing kernel particle method [11], and interpolating local Petrov-Galerkin method [12], have been presented to solve the potential and elasticity problems. In these meshless Galerkin methods, background grids are applied in numerical integration for forming the stiffness matrices.

Collocation method without element division and numerical integration is a truly meshless method. The collocation method has been widely applied to the field of engineering numerical calculation [13-18]. Spectral collocation method (pseudospectral method) based on the characteristic polynomial interpolation $[15,16]$ is a highprecision numerical method that achieves high accuracy with fewer nodes. Differential quadrature method (DQM) is also a high-precision collocation method [17, 18]. DQM applies the weighted sum of the unknown function value in the 
calculating nodes to approximate the derivative value of the unknown function. In two-dimensional problem, spectral collocation method and differential quadrature method adopt the tensor product to construct the approximation functions in rectangular domain. They cannot be directly applied to numerical calculation in geometrically complex regions. Collocation method based on radial basis function interpolation $[19,20]$ can be directly applied in complex areas. The calculation precision of this method depends on the selection of interpolation parameters that can be obtained only from a great deal of numerical calculation experience.

Embedding an irregular domain into a regular region, such as rectangular and disk, it is an effective method for solving boundary value problems of partial differential equation in complex regions [21-23]. We adopt the regular region collocation method to accurately solve the potential problems in irregular multiply connected domain by embedding the complex doubly connected domain into the regular region with polar coordinates (annular region). The barycentric Lagrange interpolation [24], a stabilized and high-precision interpolation method, is applied to discretize complex region boundary conditions. In the regular region, the barycentric interpolation collocation method $[25,26]$ is used to solve Poisson equations with Dirichlet boundary conditions.

The paper is organized as follows. In Section 2, we present the computational modeling and formulations of regular domain collocation. In Section 3, some numerical examples are given to illustrate the numerical accuracy of the proposed method, and in Section 4 we draw conclusions.

\section{Computational Modeling and Formulations}

2.1. Computational Modeling. Consider the potential problem on irregular domain as shown in Figure 1. The doubly connected domain $\Omega=\left\{(r, \theta): r_{1}(\theta) \leq r \leq r_{2}(\theta), 0 \leq \theta \leq\right.$ $2 \pi\}$ is composed of the two closed curves $\Gamma_{1}: r=r_{1}(\theta)$ and $\Gamma_{2}: r=r_{2}(\theta)(0 \leq \theta \leq 2 \pi)$.

In system of polar coordinates, the governing equation and boundary conditions of the potential problem are the following:

$$
\begin{gathered}
\Delta u=u_{r r}+\frac{1}{r} u_{r}+\frac{1}{r^{2}} u_{\theta \theta}=f(r, \theta), \quad(r, \theta) \in \Omega, \\
u\left(r_{1}, \theta\right)=g_{1}(\theta), \quad 0 \leq \theta \leq 2 \pi, \\
u\left(r_{2}, \theta\right)=g_{2}(\theta), \quad 0 \leq \theta \leq 2 \pi .
\end{gathered}
$$

In the numerical calculation, the domain $\Omega$ is embedded into the annular region composed of circumference $\Gamma_{3}: r=a$ and $\Gamma_{4}: r=b$ as shown in Figure 1. In general, let $a=$ $\min _{0 \leq \theta \leq 2 \pi}\left\{r_{1}(\theta)\right\}$ and $b=\max _{0 \leq \theta \leq 2 \pi}\left\{r_{2}(\theta)\right\}$.

2.2. Computational Formulations. Given a function $v(x)$ defined on the interval $[c, d]$ and discrete nodes $c=x_{1}<x_{2}<$ $\cdots<x_{n}=d$, the function values on the nodes are $v_{i}=v\left(x_{i}\right)$,

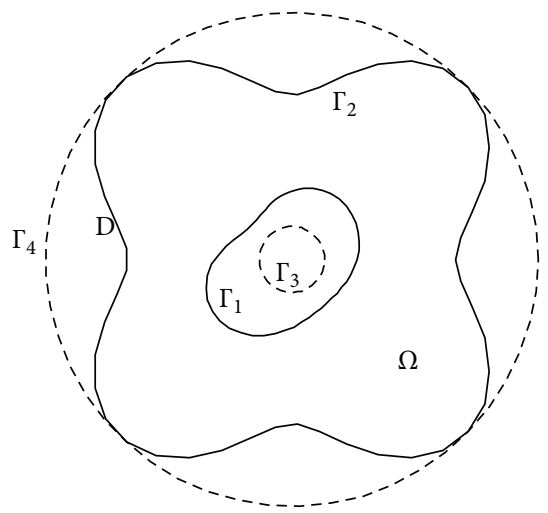

FIGURE 1: The doubly connected domain and its regular domain.

$i=1,2, \ldots, n$. The barycentric Lagrange interpolation of the function $v(x)$ on the nodes is [24]

$$
v(x)=\frac{\sum_{j=1}^{n}\left(w_{j} /\left(x-x_{j}\right)\right) v_{j}}{\sum_{j=1}^{n}\left(w_{j} /\left(x-x_{j}\right)\right)},
$$

where $w_{j}=1 / \prod_{j \neq k}\left(x_{j}-x_{k}\right), j=0,1, \ldots, n$, is barycentric interpolation weight.

The barycentric Lagrange interpolation basis function is defined as follows:

$$
L_{k}(x)=\frac{w_{k} /\left(x-x_{k}\right)}{\sum_{j=0}^{n}\left(w_{j} /\left(x-x_{j}\right)\right)}, \quad k=1,2, \ldots, n .
$$

Then, barycentric Lagrange interpolation of the function $v(x)$ can be written as

$$
v(x)=\sum_{j=1}^{n} L_{j}(x) v_{j}
$$

The $m$ th order derivative of function $v(x)$ can be expressed as

$$
v^{(m)}(x):=\frac{d^{m} v(x)}{d x^{m}}=\sum_{j=1}^{n} L_{j}^{(m)}(x) v_{j} .
$$

So the $m$ th order derivative of function $v(x)$ in the nodes $x_{1}, x_{2}, \ldots, x_{n}$ is

$$
v^{(m)}\left(x_{i}\right)=\sum_{j=1}^{n} L_{j}^{(m)}\left(x_{i}\right) v_{j}=\sum_{j=1}^{n} C_{i j}^{(m)} v_{j}, \quad i=1,2, \ldots, n,
$$

where $C_{i j}^{(m)}=L_{j}^{(m)}\left(x_{i}\right)$ indicates the $m$ th order derivative value of the $j$ th basis function at $i$ th node. Deriving (5) with respect to $x$ directly, we have

$$
\begin{gathered}
C_{i j}^{(1)}=\frac{w_{j} / w_{i}}{x_{i}-x_{j}}, \quad j \neq i, \\
C_{i i}^{(1)}=-\sum_{j=1, j \neq i}^{n} C_{i j}^{(1)} .
\end{gathered}
$$


And the entries $C_{i j}^{(m)}$ can be derived from the following recursion $[25,26]$ :

$$
\begin{gathered}
C_{i j}^{(m)}=m\left(C_{i i}^{(m-1)} C_{i j}^{(1)}-\frac{C_{i j}^{(m-1)}}{x_{i}-x_{j}}\right), \quad i \neq j, \\
C_{i i}^{(m)}=-\sum_{j=1, j \neq i}^{n} C_{i j}^{(m)} .
\end{gathered}
$$

As a result, (8) can be written in the following matrix form:

$$
\mathbf{v}^{(m)}=\mathbf{C}^{(m)} \mathbf{v} .
$$

In (11), $\mathbf{v}^{(m)}=\left[v_{1}^{(m)}, v_{2}^{(m)}, \ldots, v_{n}^{(m)}\right]^{T}$ and $\mathbf{v}=\left[v_{1}, v_{2}, \ldots, v_{n}\right]^{T}$ represent the column vector of $m$ th order derivative value and the value of the function $v(x)$ in the nodes, respectively. Matrix $\mathbf{C}^{(m)}$ is named as the $m$ th order differentiation matrix of barycentric Lagrange interpolation on nodes $x_{1}, x_{2}, \ldots, x_{n}$.

Embedding the irregular computational region $\Omega$ into a two-dimensional annular domain $D=\{(r, \theta): a \leq r \leq b, 0 \leq$ $\theta \leq 2 \pi\}, \Omega \subset D$, let $m$ and $n$ distinct computational nodes $a=r_{1}<r_{2}<\cdots<r_{m}=b$ and $0=\theta_{1}<\theta_{2}<\cdots<$ $\theta_{n}=2 \pi$ be given in the direction of $r$ and $\theta$, respectively. We can obtain $m \times n$ computational nodes of tensor product type, $\left(r_{i}, \theta_{j}\right), i=1,2, \ldots, m, j=1,2, \ldots, n$, on the domain $D$. The denoted values of function $u(r, \theta)$ in the computational nodes are $u_{i j}=u\left(r_{i}, \theta_{j}\right), i=1,2, \ldots, m, j=1,2, \ldots, n$.

The computational nodes and function values can be formed into three $m \times n$-dimensional column vectors as follows:

$$
\begin{gathered}
\mathbf{R}=\left[r_{1}, \ldots, r_{1}, r_{2}, \ldots, r_{2}, \ldots, r_{m}, \ldots, r_{m}\right]^{T}, \\
\Theta=\left[\theta_{1}, \ldots, \theta_{n}, \theta_{1}, \ldots, \theta_{n}, \ldots, \theta_{1}, \ldots, \theta_{n}\right]^{T}, \\
\mathbf{U}=\left[u_{11}, \ldots, u_{1 n}, u_{21}, \ldots, u_{2 n}, \ldots, u_{m 1}, \ldots, u_{m n}\right]^{T} .
\end{gathered}
$$

The barycentric Lagrange interpolation of the function $u(r, \theta)$ in the computational nodes can be expressed as

$$
u(r, \theta)=\sum_{i=1}^{m} \sum_{j=1}^{n} L_{i}(r) M_{j}(\theta) u_{i j},
$$

where $L_{i}(r), M_{j}(\theta)$ are barycentric Lagrange interpolation basis function on the nodes $r_{1}, r_{2}, \ldots, r_{m}$ and $\theta_{1}, \theta_{2}, \ldots, \theta_{n}$, respectively.

From formula (13), $(l+k)$ th partial derivative of the function $u(r, \theta)$ with respect to $r$ and $\theta$ can be expressed as

$$
\frac{\partial^{l+k} u}{\partial^{l} r \partial^{k} \theta}=\sum_{i=1}^{m} \sum_{j=1}^{n} L_{i}^{(l)}(r) M_{j}^{(k)}(\theta) u_{i j}, \quad l, k=0,1,2, \ldots
$$

The value of partial derivative in the computational nodes is

$$
\begin{array}{r}
u_{p q}^{(l+k)}:=\frac{\partial^{l+k} u\left(r_{p}, \theta_{q}\right)}{\partial^{l} r \partial^{k} \theta}=\sum_{i=1}^{m} \sum_{j=1}^{n} L_{i}^{(l)}\left(\theta_{q}\right) u_{i j}, \\
p=1,2, \ldots, m ; \quad q=1,2, \ldots, n .
\end{array}
$$

By using the symbols of barycentric interpolation differential matrix $\mathbf{C}^{(l)}, \mathbf{D}^{(k)}$ and matrix tensor product, “ $\otimes$ ”, formula (15) can be rewritten as the matrix form

$$
\mathbf{U}^{(l+k)}=\left(\mathbf{C}^{(l)} \otimes \mathbf{D}^{(k)}\right) \mathbf{U},
$$

where $\mathbf{C}^{(l)}, \mathbf{D}^{(k)}$ indicate $l$ th and $k$ th order barycentric Lagrange interpolation differential matrix in the nodes $r_{1}, r_{2}, \ldots, r_{m}$ and $\theta_{1}, \theta_{2}, \ldots, \theta_{n}$, respectively. Denote $\mathbf{C}^{(0)}=$ $\mathbf{I}_{m}, \mathbf{D}^{(0)}=\mathbf{I}_{n} . \mathbf{I}_{m}, \mathbf{I}_{n}$ are $m$ th and $n$th order identity matrix, respectively. $\mathbf{U}^{(l+k)}$ is $m \times n$-dimensional column vectors which is consisted of the values of partial derivative $u_{i j}^{(l+k)}=$ $\partial^{l+k} u\left(r_{i}, \theta_{j}\right) / \partial^{l} r \partial^{k} \theta$ in the nodes $\left(r_{i}, \theta_{j}\right), i=1,2, \ldots, m, j=$ $1,2, \ldots, n$,

$$
\begin{gathered}
\mathbf{U}^{(l+k)}=\left[u_{11}^{(l+k)}, \ldots, u_{1 n}^{(l+k)}, u_{21}^{(l+k)}, \ldots, u_{2 n}^{(l+k)}, \ldots,\right. \\
\left.u_{m 1}^{(l+k)}, \ldots, u_{m n}^{(l+k)}\right]^{T}
\end{gathered}
$$

By formula (16), the discrete formula of the Poisson equation (1) can be rewritten as

$$
\left[\left(\mathbf{C}^{(2)} \otimes \mathbf{I}_{n}\right)+\left(\frac{1}{\mathbf{R}}\right)\left(\mathbf{C}^{(1)} \otimes \mathbf{I}_{n}\right)+\left(\frac{1}{\mathbf{R}^{2}}\right)\left(\mathbf{I}_{m} \otimes \mathbf{D}^{(2)}\right)\right] \mathbf{U}=\mathbf{F} .
$$

Here, $1 / \mathbf{R}=\operatorname{diag}\left(1 / r_{1}, 1 / r_{2}, \ldots, 1 / r_{m}\right) \otimes \mathbf{I}_{n}, 1 / \mathbf{R}^{2}=$ $\operatorname{diag}\left(1 / r_{1}^{2}, 1 / r_{2}^{2}, \ldots, 1 / r_{m}^{2}\right) \otimes \mathbf{I}_{n}$; diag is diagonal matrix which is consisted of vector; $\mathbf{F}=f(\mathbf{r}, \boldsymbol{\theta})$. Equation (18) can be simplified into

$$
\mathbf{L U}=\mathbf{F} .
$$

The boundary conditions (2)-(3) can be discretized by barycentric Lagrange interpolation (13). We arrange $m_{1}, m_{2}$ points $\left(r_{i}^{b 1}, \theta_{i}^{b 1}\right), i=1,2, \ldots, m_{1},\left(r_{i}^{b 2}, \theta_{i}^{b 2}\right), i=1,2, \ldots, m_{2}$, on the boundaries $\Gamma_{1}, \Gamma_{2}$, respectively. In general, we need $m_{1} \geq \max (m, n), m_{2} \geq \max (m, n)$. Hence, boundary conditions (2)-(3) can be interpolated as follows:

$$
\begin{array}{r}
u\left(r_{i}^{b 1}, \theta_{i}^{b 1}\right)=\sum_{i=1}^{m} \sum_{j=1}^{n} L_{i}\left(r_{i}^{b 1}\right) M_{j}\left(\theta_{i}^{b 1}\right) u_{i j}=g_{1}\left(\theta_{i}^{b 1}\right), \\
i=1,2, \ldots, m_{1}, \\
u\left(r_{i}^{b 2}, \theta_{i}^{b 2}\right)=\sum_{i=1}^{m} \sum_{j=1}^{n} L_{i}\left(r_{i}^{b 2}\right) M_{j}\left(\theta_{i}^{b 2}\right) u_{i j}=g_{2}\left(\theta_{i}^{b 2}\right), \\
i=1,2, \ldots, m_{2} .
\end{array}
$$

Formula (20) can be simplified into

$$
\mathbf{B}_{1} \mathbf{U}=\mathbf{G}_{1}, \quad \mathbf{B}_{2} \mathbf{U}=\mathbf{G}_{2} .
$$

For numerical analysis in doubly connected domain, we need some additional conditions to ensure the singlevaluedness and smoothness of function $u(r, \theta)$. The additional conditions can be expressed as follows:

$$
u(r, 0)=u(r, 2 \pi), \quad u_{\theta}(r, 0)=u_{\theta}(r, 2 \pi) .
$$


The first and second conditions in (22) guarantee the single-valuedness and smoothness of function $u(r, \theta)$, respectively. Defining two index sets $S_{0}=\left\{k: \Theta_{k}=0\right\}, S_{1}=\{k$ : $\left.\Theta_{k}=2 \pi\right\}$, let $\mathbf{I}_{m n}^{S_{i}}$ represent a matrix whose rows come from the rows of the $(m \times n)$ order identity matrix in accordance with the index set $S_{i}(i=1,2)$; that is, $\mathbf{I}_{m n}^{S_{i}}=\mathbf{I}_{m n}\left(S_{i},:\right)$, and $\mathbf{P}_{m n}^{S_{i}}$ denote a matrix whose rows extract from the rows of the $(m \times n)$ matrix $\mathbf{I}_{m} \otimes \mathbf{D}^{(1)}$, in accordance with the index set $S_{i}(i=1,2)$. Using notations defined as above, the additional conditions (22) can be discretized as follows:

$$
\left(\mathbf{I}_{m n}^{S_{0}}-\mathbf{I}_{m n}^{S_{1}}\right) \mathbf{U}=0, \quad\left(\mathbf{P}_{m n}^{S_{0}}-\mathbf{P}_{m n}^{S_{1}}\right) \mathbf{U}=0 .
$$

Equation (23) can be rewritten as

$$
\mathbf{A}_{1} \mathbf{U}=0, \quad \mathbf{A}_{2} \mathbf{U}=0 .
$$

Owing to the additional conditions discretized on the computational nodes, we can use replacement method to apply the additional conditions [25-27]. The rows with index set $S_{0}$ in matrix $\mathbf{L}$ in (19) are replaced by rows of matrix $\mathbf{A}_{1}$, and components with index set $S_{0}$ in vector $\mathbf{F}$ are set to be zeros. The rows with index set $S_{1}$ in matrix $\mathbf{L}$ in (19) are replaced by rows of matrix $\mathbf{A}_{2}$, and components with index set $S_{1}$ in vector $\mathbf{F}$ are set to be zeros. Then, (19) modifies as

$$
\widetilde{\mathbf{L}} \mathbf{U}=\widetilde{\mathbf{F}} \text {. }
$$

Combining (25) and (21), we obtain a saddle point system:

$$
\left(\begin{array}{c}
\tilde{\mathbf{L}} \\
\mathbf{B}_{1} \\
\mathbf{B}_{2}
\end{array}\right) \mathbf{U}=\left(\begin{array}{c}
\widetilde{\mathbf{F}} \\
\mathbf{G}_{1} \\
\mathbf{G}_{2}
\end{array}\right) \text {. }
$$

Using least square method to solve (26), the function value will be gained on the regular domain $D$. Then, barycentric Lagrange interpolation formula (13) is used to compute the function value in any points on the complex region $\Omega$.

\section{Numerical Results}

In this section, we present some numerical experiments to verify the methods developed in the earlier sections. The method is validated by employing exact solutions with known boundary conditions and evaluating the computational errors. The computational programs compile using Matlab. The overconstrained equation (22) is solved by backslash operator, "\", in Matlab.

In numerical analysis, the second kind Chebyshev points on the interval $[-1,1][26,27], t_{k}=-\cos (k \pi / n), k=$ $0,1,2, \ldots, n$, are adopted as type of computational nodes. Let $x=(b+a) / 2+t(b-a) / 2$; then, the Chebyshev point $t_{k}$ on the interval $[-1,1]$ can be transformed as computational nodes $x_{k}$ on the arbitrary interval $[a, b]$. For the assessment of computational accuracy and the beauty of drawing, 1000 points are arranged on the domain $\Omega$ and their function value is calculated by the barycentric Lagrange interpolation. The absolute error and relative error of numerical computation are defined, respectively, as

$$
E_{a}=\left\|\mathbf{U}^{c}-\mathbf{U}^{e}\right\|_{\infty}, \quad E_{r}=\frac{\left\|\mathbf{U}^{c}-\mathbf{U}^{e}\right\|_{2}}{\left\|\mathbf{U}^{e}\right\|_{2}},
$$

TABLE 1: Computational error of regular domain collocation method under different number of nodes in Example 1.

\begin{tabular}{llll}
\hline$m$ & $n$ & Absolute error & Relative error \\
\hline 9 & 15 & $8.5738 \times 10^{-4}$ & $4.9203 \times 10^{-6}$ \\
9 & 21 & $8.5738 \times 10^{-4}$ & $4.9203 \times 10^{-6}$ \\
11 & 15 & $6.5263 \times 10^{-5}$ & $3.4685 \times 10^{-7}$ \\
11 & 21 & $6.5263 \times 10^{-5}$ & $3.4685 \times 10^{-7}$ \\
15 & 15 & $4.3571 \times 10^{-7}$ & $2.1605 \times 10^{-9}$ \\
19 & 15 & $4.1151 \times 10^{-9}$ & $1.6389 \times 10^{-11}$ \\
23 & 15 & $4.0905 \times 10^{-9}$ & $7.5697 \times 10^{-12}$ \\
31 & 15 & $6.2059 \times 10^{-9}$ & $1.6639 \times 10^{-11}$ \\
\hline
\end{tabular}

where $\mathbf{U}^{c}, \mathbf{U}^{e}$ are vectors of numerical computational values and analytical solution, respectively.

Example 1. Consider the doubly connected domain composed of two concentric circles. The radii of internal and external circles are $r_{1}=0.5$ and $r_{2}=2$, respectively. This is a regular domain. Boundary conditions are determined by analytical solution $u(r, \theta)=50+50 \ln r / \ln 2$. In this case, $f(r, \theta)=0$.

The absolute error and relative error with the different number of computational nodes in radial and ring direction are listed in Table 1. Due to the fact that the solution is independent of the variable $\theta$, the nodes number in ring direction does not affect the computational errors. It can be seen from Table 1 that when the nodes number in radius direction is increased, the computational errors are decreased. Figure 2(a) shows the relations of nodes number and computational errors with Chebyshev points. For comparison, we solve this problem under equidistant points, whose relations of nodes number and computational errors are shown in Figure 2(b). It can be seen from Figure 2 that the numerical accuracy of the Chebyshev points is higher than the equidistant points. When the nodes number is larger, we find that the solution is unstable using equidistant nodes.

Example 2. Consider the doubly connected domain composed of the kite external curve $r=$ $\sqrt{(0.6 \cos \theta+0.3 \cos 2 \theta-0.2)^{2}+(0.6 \sin \theta)^{2}}$ and the bean internal curve $r=(0.5+0.2 \cos \theta+0.1 \sin 2 \theta) /(1.5+0.7 \cos \theta)$. The radii of internal and external circles are $a=0.2$ and $b=0.9$, respectively, as shown in Figure 3 .

Boundary conditions are determined by analytical solution $u(r, \theta)=r^{2} \cos 2 \theta$. In this case, $f(r, \theta)=0$. On regular region and doubly connected domain, the distribution of the compute nodes is shown in Figure 4.

The absolute error and relative error with the different number of computational nodes in radial and ring direction are listed in Table 2. From Table 2, adopting 9 radial nodes and 31 circular nodes, the absolute error and relative error reach $10^{-12}$ orders of magnitude. The calculation accuracy of the proposed method is very excellent. If we increase the number of computational nodes, the calculation accuracy still remains the high precision. The distribution of absolute error 


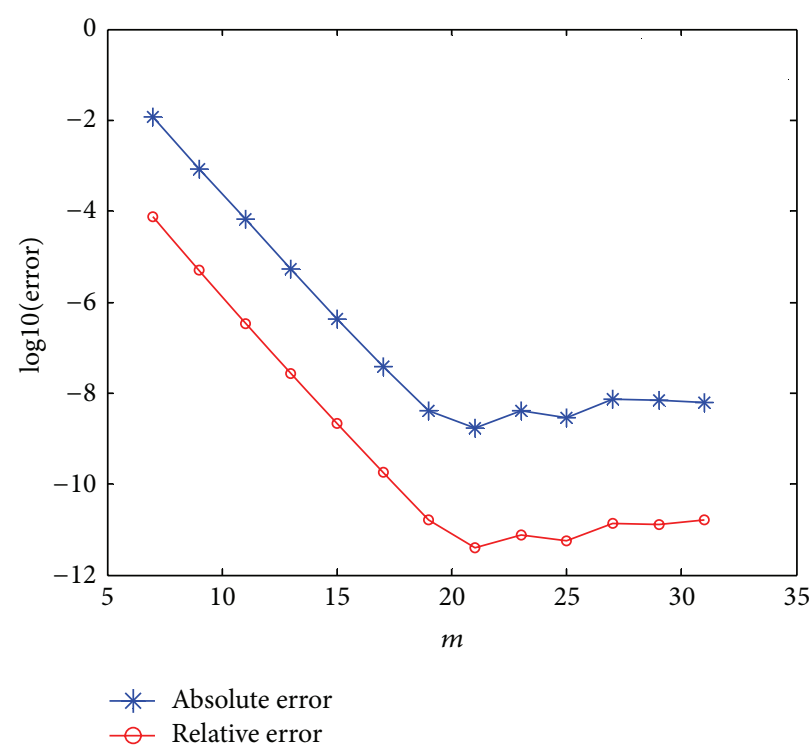

(a)

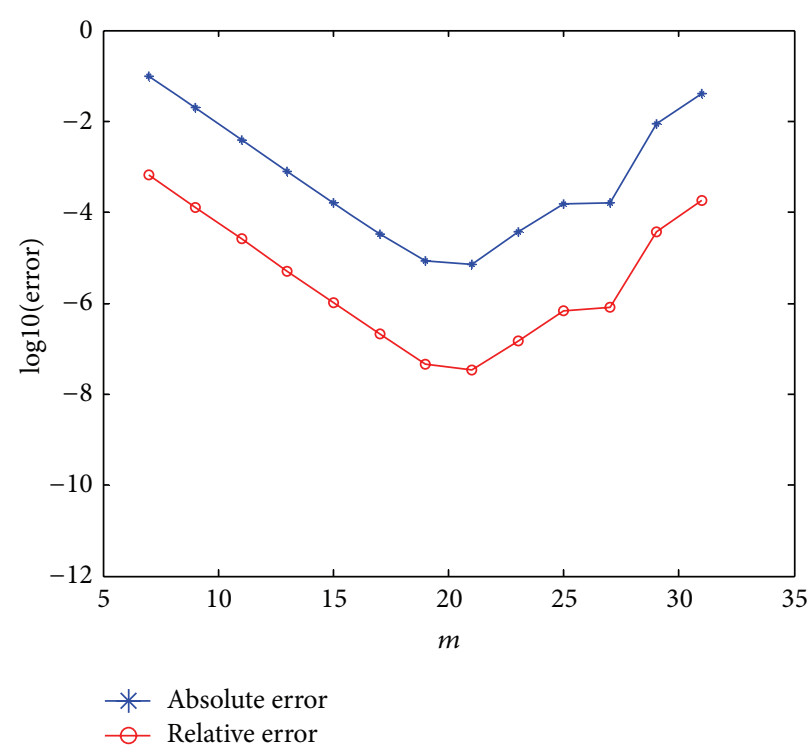

(b)

FIGURE 2: The relations of nodes number and computational errors with different types of nodes. (a) Chebyshev point; (b) equidistant nodes.

TABLE 2: Computational error of regular domain collocation method under different number of nodes in Example 2.

\begin{tabular}{llll}
\hline$m$ & $n$ & Absolute error & Relative error \\
\hline 9 & 15 & $1.4678 \times 10^{-3}$ & $8.2523 \times 10^{-4}$ \\
9 & 21 & $8.5082 \times 10^{-8}$ & $4.6690 \times 10^{-8}$ \\
9 & 31 & $7.0509 \times 10^{-12}$ & $3.6625 \times 10^{-12}$ \\
9 & 41 & $2.1288 \times 10^{-11}$ & $1.0692 \times 10^{-11}$ \\
11 & 15 & $9.2872 \times 10^{-4}$ & $5.8960 \times 10^{-4}$ \\
11 & 21 & $2.6053 \times 10^{-7}$ & $1.3558 \times 10^{-7}$ \\
11 & 31 & $7.0704 \times 10^{-11}$ & $3.0779 \times 10^{-11}$ \\
11 & 41 & $5.400 \times 10^{-11}$ & $3.1968 \times 10^{-11}$ \\
\hline
\end{tabular}

on the computing node is shown in Figure 5. Figure 6 depicts the image of numerical solutions on irregular domain.

In another highly accurate numerical method, the collocation Trefftz method, the absolute error of $u$ is $10^{-12}$ orders of magnitude through 546 iterations under parameter $m=15$ and a stopping criterion $10^{-15}$ [14]. The numerical precision of the proposed method in this paper is the same as the highly accurate collocation Trefftz method.

Example 3. Consider the doubly connected domain composed of the epitrochoid external curve $r=\sqrt{26-10 \cos 4 \theta}$ and the bean internal curve $r=(2.5+\cos \theta+0.5 \sin 2 \theta) /(1.5+$ $0.7 \cos \theta)$. The radii of internal and external circles are $a=$ 1.25 and $b=6$, respectively, as shown in Figure 7.

Boundary conditions are determined by analytical solution $u(r, \theta)=e^{r \cos \theta} \cos (r \sin \theta)$. In this case, $f(r, \theta)=0$. Adopting 16 radial nodes and 61 circular nodes, the absolute error and relative error are $1.0008 \times 10^{-5}$ and $6.2942 \times 10^{-8}$, respectively. The distribution of the compute node error is

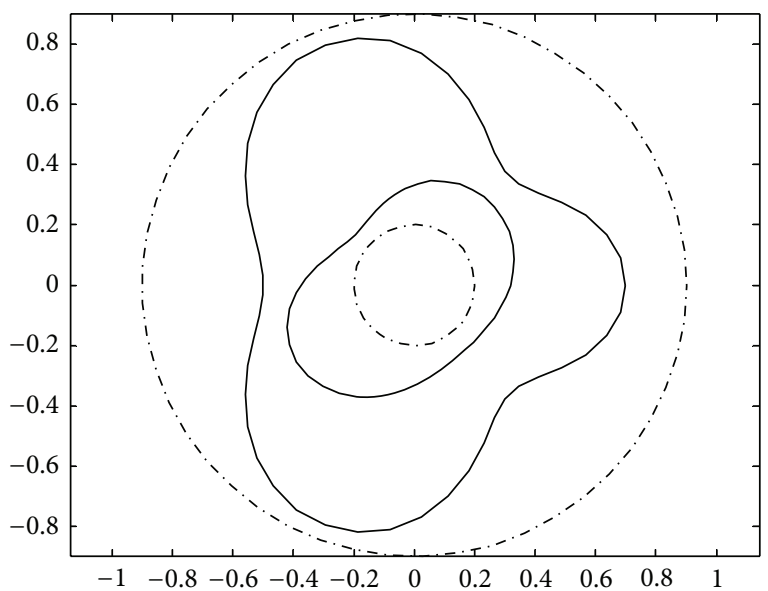

FIGURE 3: The doubly connected domain and its regular domain in Example 2.

shown in Figure 8. Figure 9 depicts the image of numerical solutions on irregular domain.

Example 4. Consider the eccentric annular region composed of the external eccentric circle $r=\cos \theta+\sqrt{\cos ^{2} \theta+21 / 4}$ and the internal circumference $r=1$. The radii of internal and external circles are $a=1$ and $b=3.5$, respectively, as shown in Figure 10.

Boundary conditions are determined by analytical solution $u(r, \theta)=r^{2}(\cos 2 \theta+\sin 2 \theta)$. In this case, $f(r, \theta)=0$. Adopting 9 radial nodes and 31 circular nodes, the absolute error and relative error reach $1.0233 \times 10^{-9}$ and $3.7942 \times$ $10^{-11}$ order of magnitude, respectively. The distribution of the compute node error is shown in Figure 11. Figure 12 depicts the image of numerical solutions on irregular domain. 


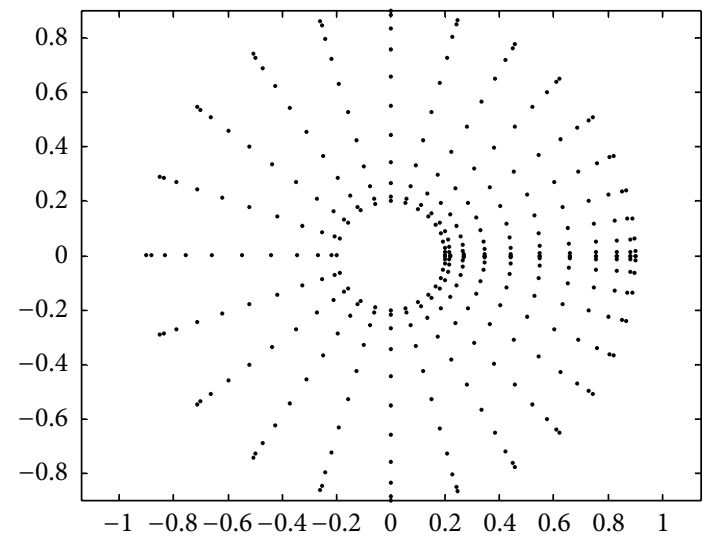

(a)

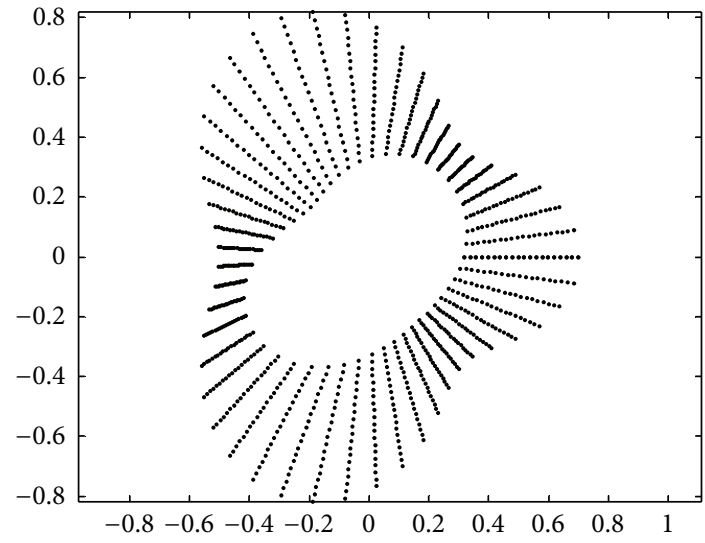

(b)

FIGURE 4: Distribution of computational nodes on regular and doubly connected domains in Example 2. (a) Distribution of computational nodes; (b) distribution of interpolating nodes in postprocessing.

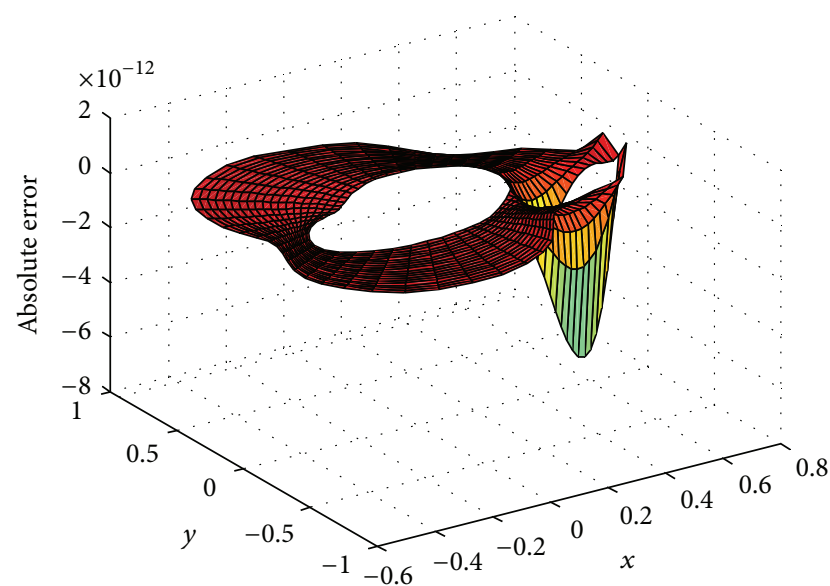

FIGURE 5: The error distribution of nodes using regular domain collocation method in Example 2.

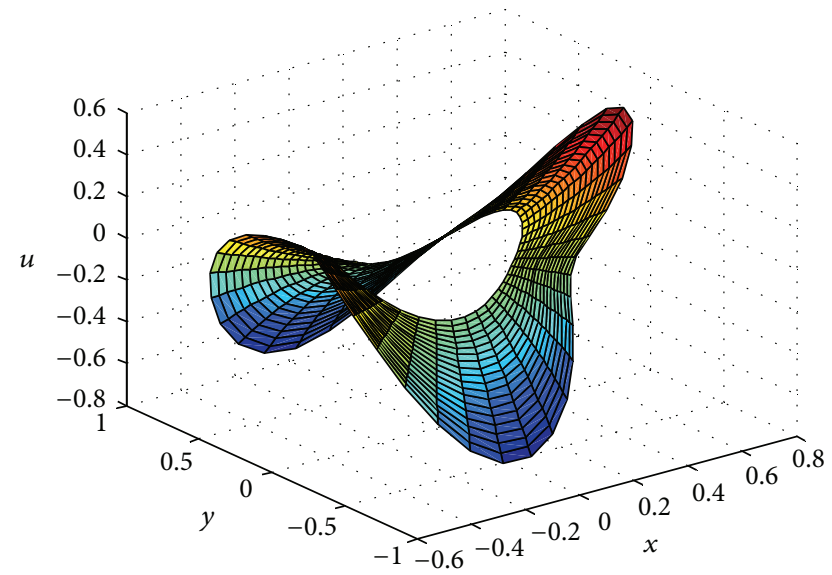

FIGURE 6: The image of numerical solutions on irregular domain in Example 2.

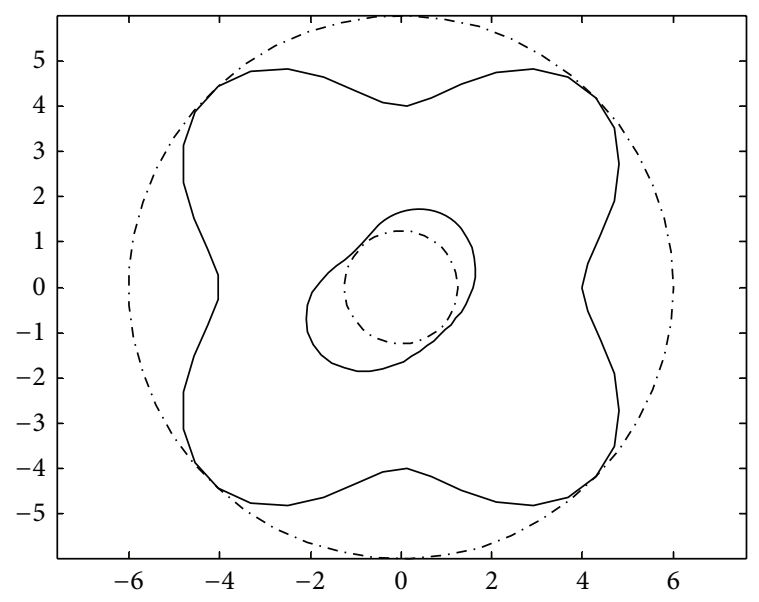

FIgURE 7: The doubly connected domain and its regular domain in Example 3.

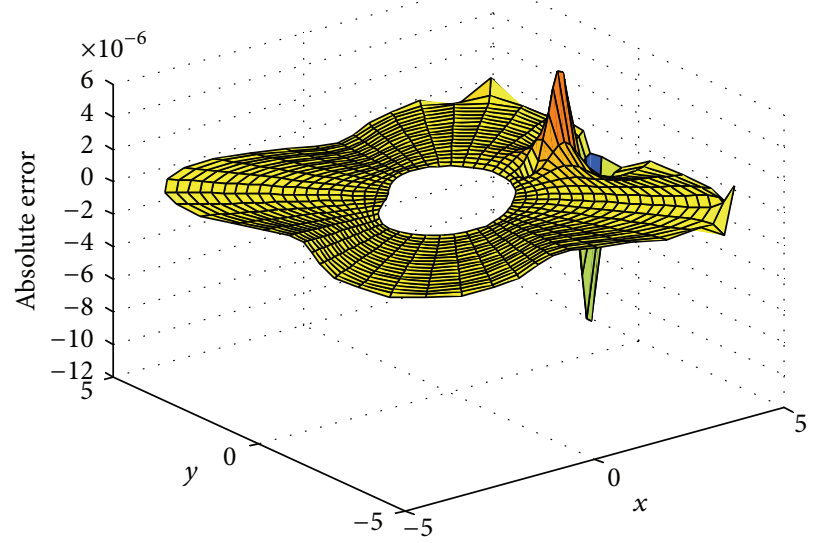

FIGURE 8: The error distribution of nodes using regular domain collocation method in Example 3. 


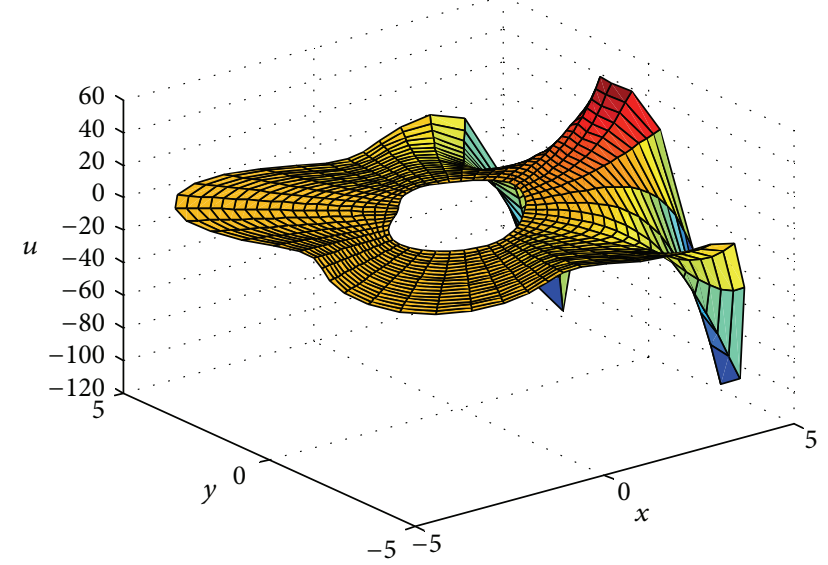

FIGURE 9: The image of numerical solutions on irregular domain in Example 3.

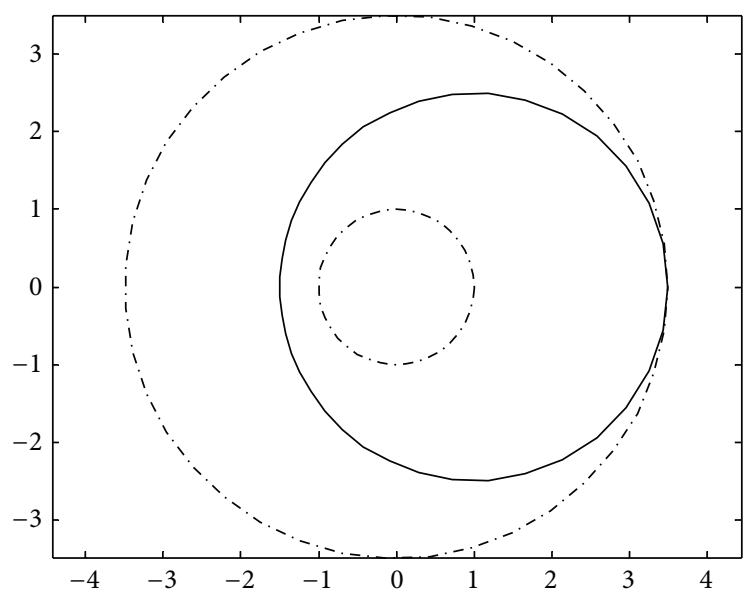

FIgURE 10: The doubly connected domain and its regular domain in Example 4.

Example 5. Consider the doubly connected domain composed of the external ellipse $x^{2} / 100+y^{2} / 64=1$ and the internal ellipse $x^{2} / 25+y^{2} / 4=1$. The radii of internal and external circles are $a=2$ and $b=10$, respectively, as shown in Figure 13.

Boundary conditions are determined by analytical solution $u(r, \theta)=4 r^{2} \cos ^{2} \theta+3 r^{2} \cos \theta \sin \theta-2 r \sin \theta+8$. In this case, $f(r, \theta)=8$. Adopting 11 radial nodes and 31 circular nodes, the absolute error and relative error reach $5.6604 \times$ $10^{-8}$ and $4.1297 \times 10^{-11}$ order of magnitude, respectively. The distribution of the compute node error is shown in Figure 14. Figure 15 depicts the image of numerical solutions on irregular domain.

\section{Conclusions}

Regular domain collocation method is an effective method to solve the potential problem on doubly connected domain of complex boundary and has the very high calculation

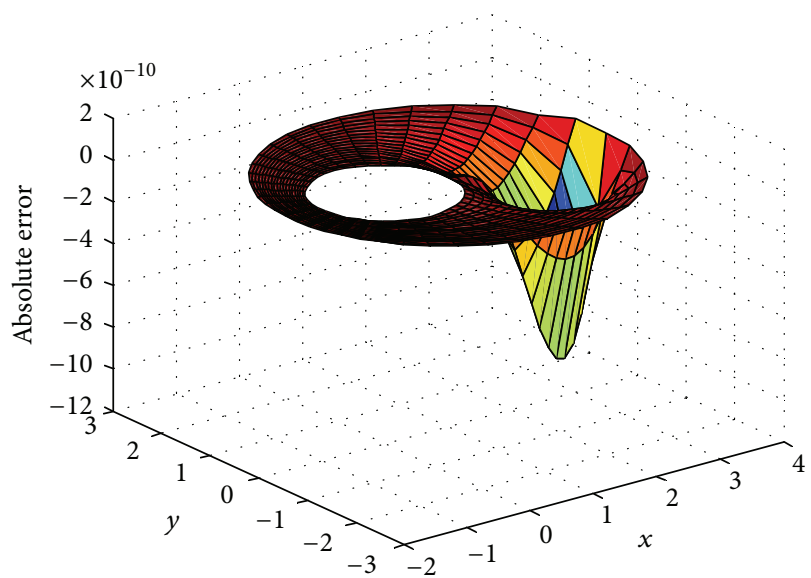

FIGURE 11: The error distribution of nodes using regular domain collocation method in Example 4.

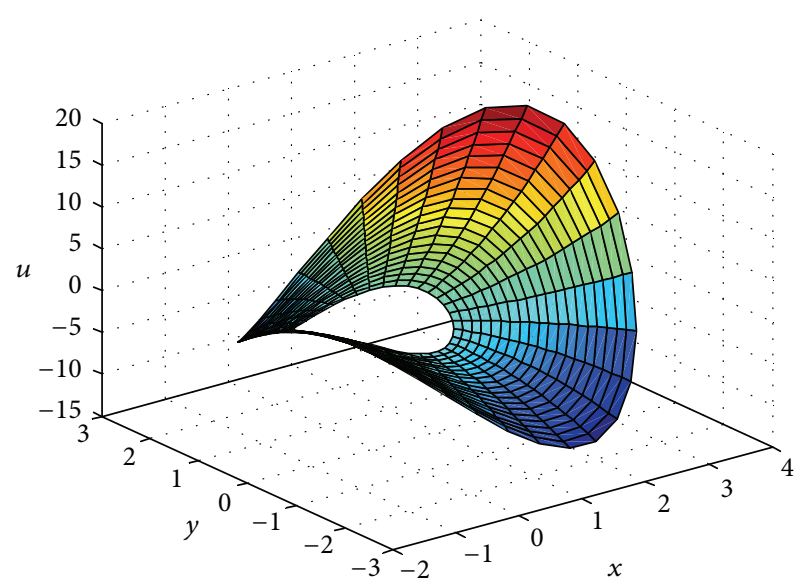

FIGURE 12: The image of numerical solutions on irregular domain in Example 4.

precision. Barycentric interpolation collocation method can be applied to solve the boundary value problem of differential equations on irregular region by regular domain collocation method. So the application scope of barycentric interpolation collocation method is expanded.

The key problem is how to discrete and impose boundary conditions in the regular domain collocation method. Using barycentric Lagrange interpolation method, a stabilized, high-precision interpolation method, we can accurately and conveniently discretize boundary conditions on irregular boundary. Numerical calculation indicates that if the boundary point is less than the maximum of the radial nodes and circular nodes, the resulting coefficient matrix of algebraic equation is not column full rank. As a result, we cannot get numerical solution.

Regular domain collocation method proposed in this paper can be directly applied to solve the differential equation boundary value problem on the irregular simply connected region. 


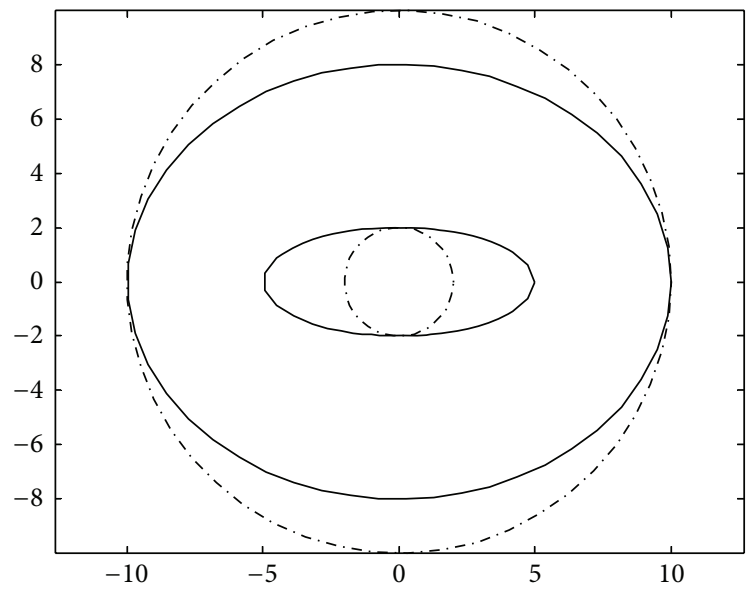

Figure 13: The doubly connected domain and its regular domain in Example 5.

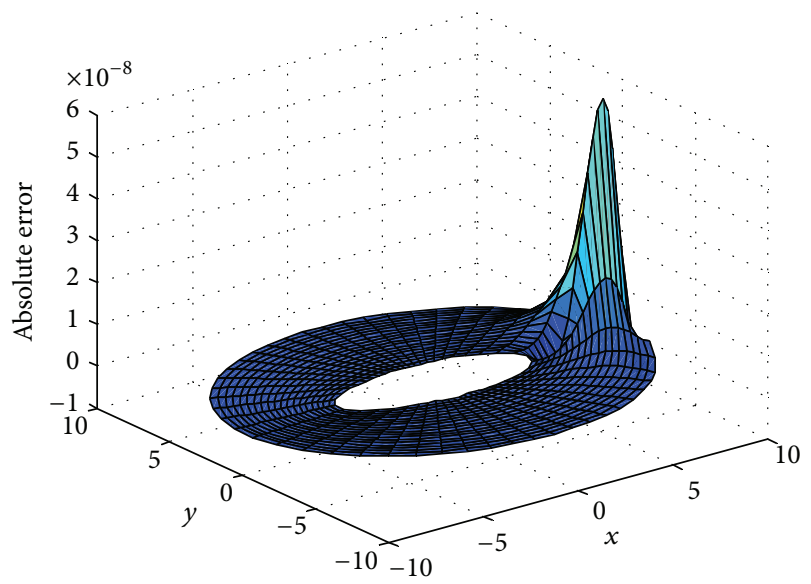

FIGURE 14: The error distribution of nodes using regular domain collocation method in Example 5.

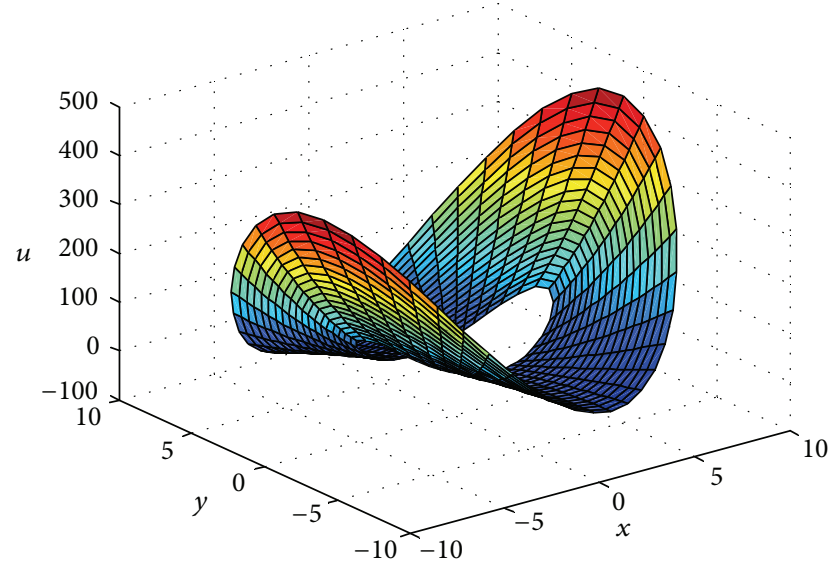

FIGURE 15: The image of numerical solutions on irregular domain in Example 5.

\section{Conflict of Interests}

The authors declare that there is no conflict of interests regarding the publication of this paper.

\section{Acknowledgments}

The authors would like to thank the support of the National Basic Research Program of China (Grant no. 2010CB732002), the National Natural Science Foundation of China (Grants nos. 51179098 and 51379113), the Specialized Research Fund for the Doctoral Program of Higher Education of China (Grant no. 20120131110031), and the Program for New Century Excellent Talents in University of Ministry of Education of China (Grant no. NCET-12-2009).

\section{References}

[1] L. G. Olson, G. C. Georgiou, and W. W. Schultz, "An efficient finite element method for treating singularities in Laplace's equation," Journal of Computational Physics, vol. 96, no. 2, pp. 391-410, 1991.

[2] Z. Cai, S. Kim, S. Kim, and S. Kong, "A finite element method using singular functions for Poisson equations: mixed boundary conditions," Computer Methods in Applied Mechanics and Engineering, vol. 195, no. 19-22, pp. 2635-2648, 2006.

[3] Y. M. Cheng, J. Wang, and R. Li, "The complex variable elementfree Galerkin (CVEFG) method for two-dimensional elastodynamics problems," International Journal of Applied Mechanics, vol. 4, no. 4, Article ID 1250042, 2012.

[4] J. Wang and Y. M. Cheng, "New complex variable meshless method for advection-diffusion problems," Chinese Physics B, vol. 22, no. 3, Article ID 030208, 2013.

[5] L. Chen, H. P. Ma, and Y. M. Cheng, "Combining the complex variable reproducing kernel particle method and the finite element method for solving transient heat conduction problems," Chinese Physics B, vol. 22, no. 5, Article ID 050202, 2013.

[6] D. M. Li, K. M. Liew, and Y. Cheng, "An improved complex variable element-free Galerkin method for two-dimensional large deformation elastoplasticity problems," Computer Methods in Applied Mechanics and Engineering, vol. 269, pp. 72-86, 2014.

[7] J. Wang, J. Wang, F. Sun, and Y. Cheng, "An interpolating boundary element-free method with nonsingular weight function for two-dimensional potential problems," International Journal of Computational Methods, vol. 10, no. 6, Article ID 1350043, 2013.

[8] Z. Zhang, J. Wang, Y. M. Cheng, and K. M. Liew, “The improved element-free Galerkin method for three-dimensional transient heat conduction problems," Science China Physics, Mechanics \& Astronomy, vol. 56, no. 8, pp. 1568-1580, 2013.

[9] Z. Zhang, S. Y. Hao, K. M. Liew, and Y. M. Cheng, "The improved element-free Galerkin method for two-dimensional elastodynamics problems," Engineering Analysis with Boundary Elements, vol. 37, no. 12, pp. 1576-1584, 2013.

[10] M. J. Peng, R. X. Li, and Y. M. Cheng, "Analyzing threedimensional viscoelasticity problems via the improved element-free Galerkin (IEFG) method," Engineering Analysis with Boundary Elements, vol. 40, pp. 104-113, 2014. 
[11] Y. J. Weng and Y. M. Cheng, "Analyzing variable coefficient advection-diffusion problems via complex variable reproducing kernel particle method," Chinese Physics B, vol. 22, no. 9, Article ID 090204, 2013.

[12] L. Chen, C. Liu, H. P. Ma, and Y. M. Cheng, "An interpolating local Petrov-Galerkin method for potential problems," International Journal of Applied Mechanics, vol. 6, no. 1, Article ID 1450009, 2014.

[13] Z.-C. Li, T.-T. Lu, H.-T. Huang, and A. H.-D. Cheng, "Trefftz, collocation, and other boundary methods-a comparison," Numerical Methods for Partial Differential Equations, vol. 23, no. 1, pp. 93-144, 2007.

[14] C.-S. Liu, "A highly accurate collocation Trefftz method for solving the Laplace equation in the doubly connected domains," Numerical Methods for Partial Differential Equations, vol. 24, no. 1, pp. 179-192, 2008.

[15] B. Bialecki and A. Karageorghis, "Spectral Chebyshev-Fourier collocation for the Helmholtz and variable coefficient equations in a disk," Journal of Computational Physics, vol. 227, no. 19, pp. 8588-8603, 2008.

[16] W. Heinrichs, "Spectral collocation schemes on the unit disc," Journal of Computational Physics, vol. 199, no. 1, pp. 66-86, 2004.

[17] Z. Zong and K. Y. Lam, "A localized differential quadrature (LDQ) method and its application to the 2D wave equation," Computational Mechanics, vol. 29, no. 4-5, pp. 382-391, 2002.

[18] T. Y. Wu, G. R. Liu, and Y. Y. Wang, "Application of the generalized differential quadrature rule to initial-boundaryvalue problems," Journal of Sound and Vibration, vol. 264, no. 4, pp. 883-891, 2003.

[19] H. Y. Hu, J. S. Chen, and W. Hu, "Weighted radial basis collocation method for boundary value problems," International Journal for Numerical Methods in Engineering, vol. 69, no. 13, pp. 2736-2757, 2007.

[20] L. Ling and R. Schaback, "An improved subspace selection algorithm for meshless collocation methods," International Journal for Numerical Methods in Engineering, vol. 80, no. 13, pp. 1623-1639, 2009.

[21] M. Buffat and L. Le Penven, "A spectral fictitious domain method with internal forcing for solving elliptic PDEs," Journal of Computational Physics, vol. 230, no. 7, pp. 2433-2450, 2011.

[22] Y. C. Zhou and G. W. Wei, "On the fictitious-domain and interpolation formulations of the matched interface and boundary (MIB) method," Journal of Computational Physics, vol. 219, no. 1, pp. 228-246, 2006.

[23] C. Veeramani, P. D. Minev, and K. Nandakumar, "A fictitious domain formulation for flows with rigid particles: a nonLagrange multiplier version," Journal of Computational Physics, vol. 224, no. 2, pp. 867-879, 2007.

[24] J.-P. Berrut and L. N. Trefethen, "Barycentric Lagrange interpolation,” SIAM Review, vol. 46, no. 3, pp. 501-517, 2004.

[25] Z. Wang, J. Qi, and B. Tang, "Numerical solution of singular source problems with barycentric interpolation," Chinese Journal of Computational Physics, vol. 28, no. 6, pp. 883-888, 2011.

[26] S. C. Li and Z. Q. Wang, The High Precision Meshless Barycentric Interpolation Method: Algorithms, Programs and Application in Engineering, Science Press, Beijing, China, 2012.

[27] L. N. Trefethen, Spectral Methods in Matlab, SIAM, Philadelphia, Pa, USA, 2000. 


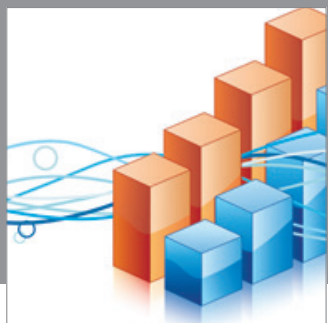

Advances in

Operations Research

mansans

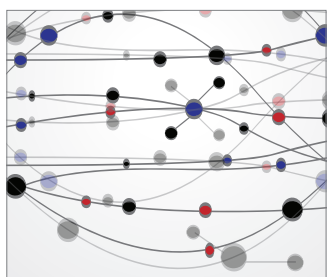

The Scientific World Journal
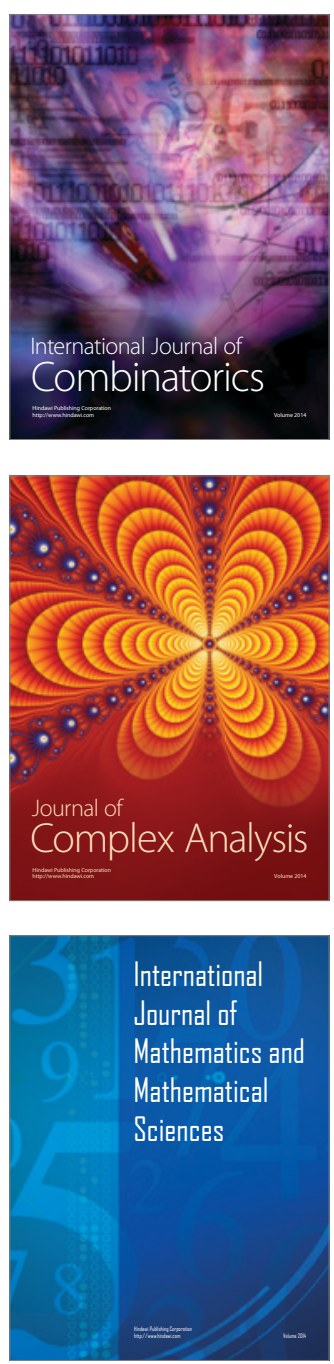
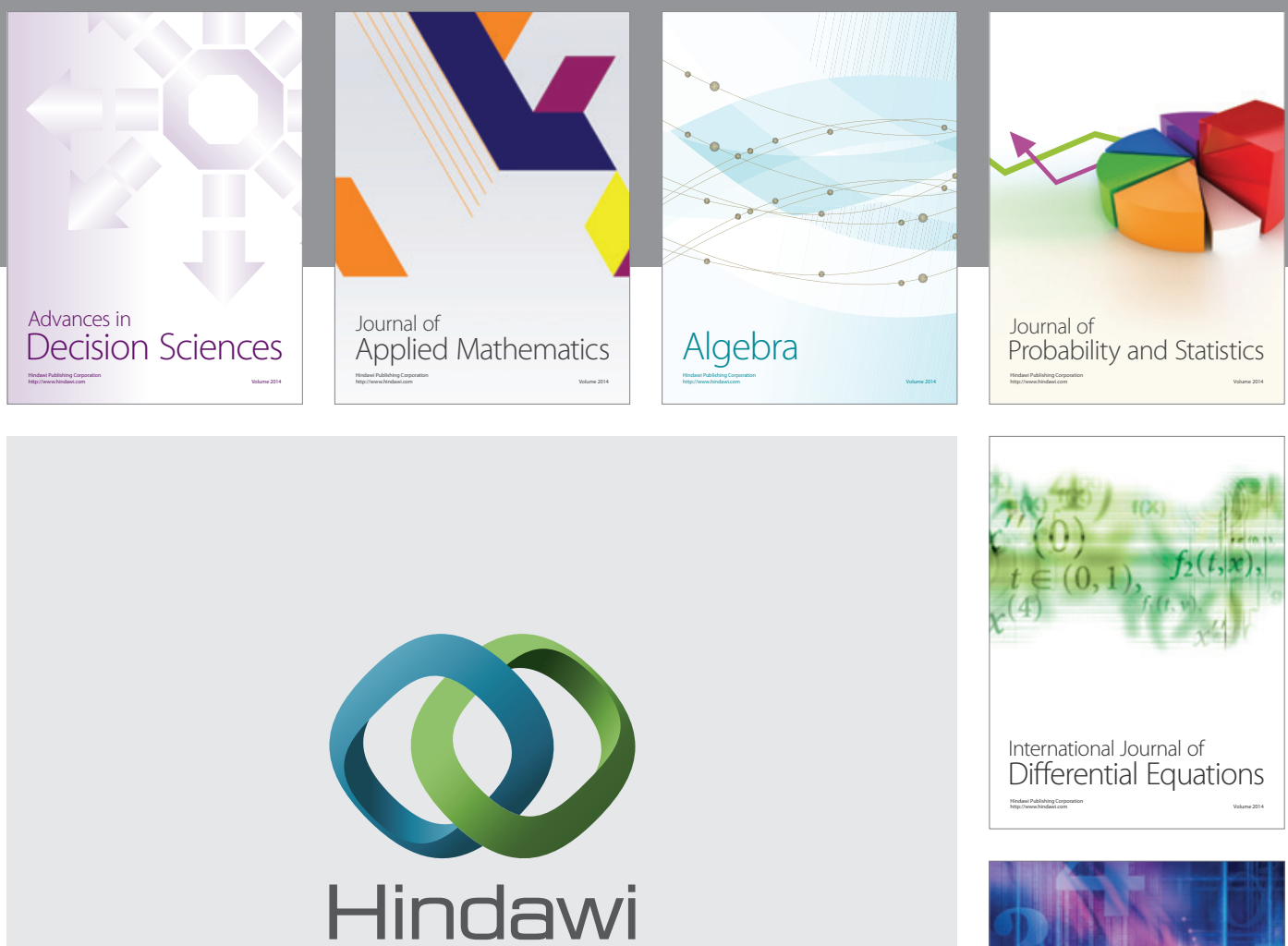

Submit your manuscripts at http://www.hindawi.com
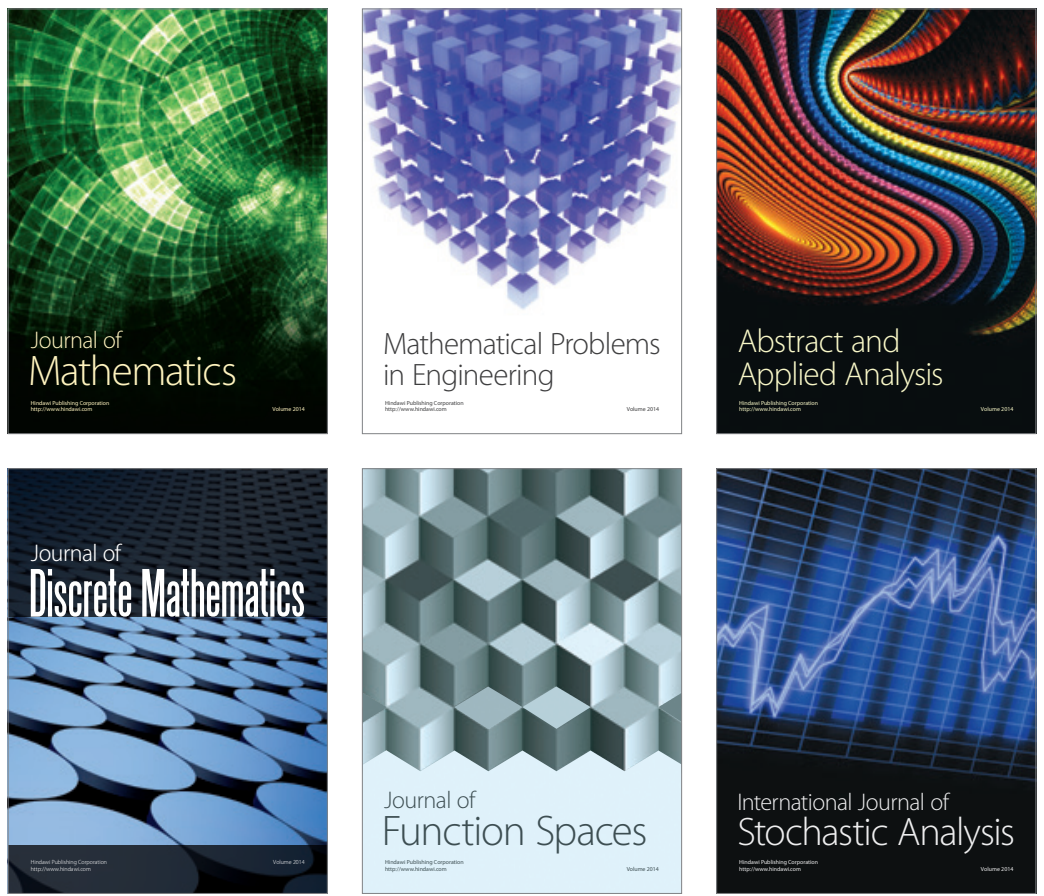

Journal of

Function Spaces

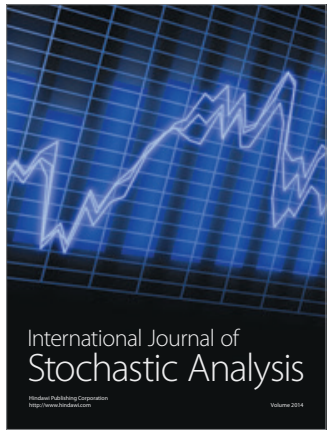

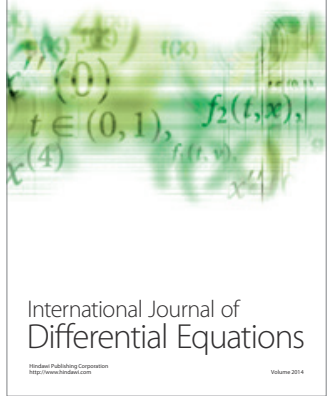
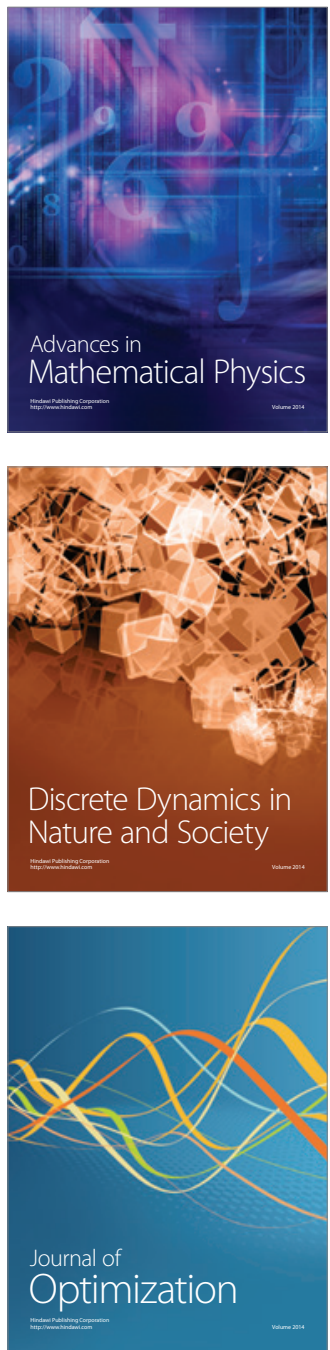